BMJ Open

Diabetes

Research

\& Care
To cite: McEwan $P$, Larsen Thorsted B, Wolden M, et al. Healthcare resource implications

of hypoglycemia-related hospital admissions and inpatient hypoglycemia: retrospective record-linked cohort studies in England. BMJ Open Diabetes Research and Care 2015:3:e000057. doi:10.1136/bmjdrc-2014000057

- Additional material is published online only. To view please visit the journal online (http://dx.doi.org/10. 1136/bmjdrc-2014-000057)

Received 27 August 2014 Revised 29 January 2015 Accepted 10 February 2015

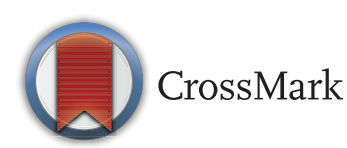

${ }^{1}$ Swansea University, Swansea, UK

${ }^{2}$ Novo Nordisk A/S, Bagsværd, Denmark ${ }^{3}$ Statcon ApS, Kokkedal, Denmark

${ }^{4}$ Llandough Hospital,

Cardiff, UK

Correspondence to Dr Phil McEwan; phil.mcewan@heor.co.uk

\title{
Healthcare resource implications of hypoglycemia-related hospital admissions and inpatient hypoglycemia: retrospective record-linked cohort studies in England
}

\author{
Philip McEwan, ${ }^{1}$ Brian Larsen Thorsted, ${ }^{2}$ Michael Wolden, ${ }^{2}$ Judith Jacobsen, ${ }^{3}$ \\ Marc Evans ${ }^{4}$
}

\section{ABSTRACT}

Objective: Using a retrospective cohort study, the mean length of hospital stay (LoS) and total per-patient expenditure for hypoglycemia requiring admission to hospital were estimated. In a separate matched retrospective cohort study, the effect of inpatient hypoglycemia on LoS, expenditure, and risk of allcause mortality while admitted was investigated. Methods: The cohorts consisted of patients aged $\geq 18$ years with a diagnosis of type 1 or 2 diabetes between January 1, 2002 and October 30, 2012 in the Clinical Practice Research Datalink database, who had initiated insulin treatment and had a recording of hypoglycemia in the same period. In the matched retrospective cohort study, exposed patients (who experienced hypoglycemia in hospital) were casematched with patients who did not experience hypoglycemia during admission (unexposed). Generalized linear regression was used to estimate LoS. Risk of all-cause mortality was evaluated via logistic regression.

Results: In the retrospective cohort study (1131 patients), mean LoS was 5.46 (95\% Cl 4.62 to 6.45$)$ days for type 1 diabetes, and $5.04(95 \% \mathrm{Cl} 4.46$ to 5.71) days for type 2 diabetes. Mean cost per admission was $£ 1034$ (95\% Cl £855 to £1253). In the matched retrospective cohort study (1079 pairs of patients), exposed patients had a mean LoS of 11.91 days $(95 \% \mathrm{Cl} 10.96$ to 12.94 days) versus 4.80 (95\% Cl 4.41 to 5.23 ) for unexposed patients, $p<0.0001$. Exposed patients had a higher mortality risk compared with unexposed patients (OR $1.439(95 \% \mathrm{Cl}$ 1.060to 1.952), $p=0.0195)$. Total average per-patient cost for exposed patients was GBP (£)2235, 40\% $(p<0.0001)$ higher than total average admission cost in unexposed patients.

Conclusions: Hypoglycemia has a significant negative impact on patient outcomes, healthcare resource use, and expenditure.

\section{RESEARCH QUESTIONS}

- What are the total national costs of treating hypoglycemia in hospital?

\section{Key messages}

- Hospitalization of people with diabetes following a hypoglycemic episode leads to significant expenditure and use of healthcare resources.

- Inpatients who experience hypoglycemia are likely to have an increased length of hospital stay and are at a higher risk of dying in hospital.

- Reducing the incidence of hypoglycemia in the community and in hospital is medically and economically important, with the potential to generate significant cost savings for healthcare providers at a national level.

- To what extent does comorbidity account for patients' risk of hypoglycemia, subsequent length of hospital stay, and risk of mortality?

- Is it possible to identify, through prescribing patterns, patients at high risk of hypoglycemia with the aim of reducing the economic burden on the healthcare system?

Hypoglycemia is one of the most common and serious side effects of insulin therapy, in some cases leading to hospitalization. Although only a small proportion of hypoglycemic episodes result in hospital admission, treating patients is a major economic burden for healthcare providers. ${ }^{1}$ Treatment costs may include general practitioner attendance, ambulance transport, accident and emergency care, and expenses incurred during the hospital stay. Among elderly patients, nearly $25 \%$ of hospital admissions have been attributed to either insulin or oral antidiabetic agents, and it has been estimated that the annual direct cost of treating severe hypoglycemic episodes could be in excess of $£ 13$ million in the UK. ${ }^{2}{ }^{3}$ Hypoglycemia is also common among inpatients with diabetes, occurring in approximately $8 \%$ of admissions, 
and leading to a substantial increase in the length of hospital stay (LoS) and mortality. ${ }^{4}$ Aside from its acute physiological effects, hypoglycemia has been associated with an elevated risk of cardiovascular events, fall-related fractures, and death, particularly among hospitalized patients, all of which add to the LoS and total cost of treatment. ${ }^{6-10}$

Notwithstanding, the resource implications of treating hypoglycemic episodes at the national level remain poorly understood. The reason for this is twofold: first, the true prevalence of hypoglycemia is unknown due to under-reporting of events; ${ }^{3}$ second, the long-term economic consequences of hypoglycemia are modeled estimates-the direct cost of a single episode being an average derived from a number of studies. ${ }^{11}{ }^{12}$ However, there is growing appreciation of the cost implications of hypoglycemia. This is in part due to recent publications that have attempted to estimate the economic burden, but also as a result of the growing population of people with diabetes who require blood glucose-lowering therapy, leading to an increase in hospital admissions resulting from hypoglycemia. ${ }^{13}$

We carried out two separate analyses: 1) a retrospective cohort study designed to estimate the mean LoS, associated risk factors, and total per-patient expenditure resulting per severe hypoglycemic episode requiring hospitalization, in patients with type 1 or 2 diabetes; 2) and a matched retrospective cohort study that estimated the marginal effect of in-hospital hypoglycemia on LoS and total per-patient expenditure in hospital admitted inpatients with type 1 or 2 diabetes. In the matched retrospective cohort study, excess risk of mortality among patients experiencing a hypoglycemic episode was also estimated.

\section{METHODS}

Data for both cohorts were obtained from the Clinical Practice Research Datalink (CPRD) database. The CPRD maintains anonymized, longitudinal primary care records from participating general practices in the UK, and represents approximately $8 \%$ of the population. ${ }^{14}$ All practices submitting data to the CPRD abide by an agreed protocol for the collection of demographic, clinical, laboratory, and prescription data.

In the present study, data from the CPRD were linked with the Hospital Episodes Statistics (HES) database. The HES database holds information on all admissions, outpatient appointments, and emergency attendances at National Health Service (NHS) hospitals in England. The study populations in the retrospective and matched retrospective cohort studies were different. Both cohorts included all patients in England with type 1 or 2 diabetes, aged $\geq 18$ years, eligible for linkage with HES, with a READ/OXMIS diagnosis code for diabetes (see online supplementary table S1) between January 1, 2002 and October 30, 2012 in the CPRD, who had initiated insulin treatment (at least two prescriptions) and had a recording of hypoglycemia in the HES database in the same period (January 1, 2002 and October 30, 2012). Hypoglycemic episodes in the HES were identified using International Classification of Disease V.10 (ICD-10) codes (see online supplementary table S1). All hypoglycemic episodes required assistance by a third party, and would therefore be classified as severe under the American Diabetes Association (ADA) guidelines. ${ }^{15}$ All patients had a READ code for diabetes type; however, to improve the accuracy of type 1 diabetes classification, the following criteria were required: READ code for type 1 diabetes, insulin treatment started a minimum of 1 year after diagnosis, patient not treated with oral antidiabetic drugs (OADs) before insulin initiation. Patients not meeting these criteria were classified as type 2 .

In the retrospective cohort, to assess the LoS, registration of hypoglycemia as the primary diagnosis was required in addition to the previously stated joint inclusion criteria. Patients were followed up from admission (due to hypoglycemia) until discharge.

In the matched retrospective cohort study-in addition to the joint inclusion criteria-to evaluate the marginal effect of inpatient hypoglycemia on LoS, total per-patient expenditure, and risk of all-cause mortality, we identified all patients who experienced hypoglycemia (exposed) during hospitalization in the study period (January 1, 2002 and October 30, 2012) and matched them in a 1:1 ratio with those who had the same primary diagnosis and diabetes type, but who did not experience hypoglycemia (unexposed). Primary diagnosis was established using 3-digit ICD-10 codes, with the exception of codes A1, N0, T5, L8, J4, and G9, which were collapsed to two digits in order to find matches. Patients with a primary diagnosis of hypoglycemia were excluded.

The following covariates were included in the analyses: gender, age, geographical region, type of diabetes, use of medication (other than insulin), body mass index (BMI), glycemic control (glycated hemoglobin, $\mathrm{HbA}_{1 \mathrm{c}}$ ), smoking status, and Charlson comorbidity index (based only on registrations in the CPRD). ${ }^{16} \mathrm{BMI}$ and $\mathrm{HbA}_{1 \mathrm{c}}$ measurements were recorded in a 6-month window before the index date. When multiple measurements were available, those closest to the index date were used. Statin and antihypertensive medication use was recorded in a 60-day window before the index date. READ/ OXMIS and ICD-10 codes for hypoglycemia, diabetes type, smoking status, and British National Formulary chapters for antihypertensive medication and statins are available in online supplementary table S1. Class of insulin (not on product level) was also recorded.

To estimate the in-hospital cost of treating patients for hypoglycemia, we combined data from the HES database with Healthcare Resource Group (HRG) 'groupers'. HRGs are groupings that consist of patient events judged to consume a similar level of resources, for example, specialist palliative care. Under this process, total hospital costs are calculated initially at the 
treatment service level (eg, wards, theatres, and pharmacy), then among specialties, and finally in HRGs. In our study, HRG groupers were used to associate inpatient procedures in the Office of Population Censuses and Surveys 4 (OPCS-4) procedural classification format with a cost. Costs were reported in £s at 2012 prices. Inpatient costs were derived from the NHS HRG Grouper Software, which combines procedure codes and ICD-10 diagnostic codes to output the most relevant HRG iso-resource code and associated tariff. We used the most up-to-date grouper tariffs (HRG4+ 2011/2012) in order to reflect the current levels of resource use for severe hypoglycemia. ${ }^{17}$ Using this methodology, we were able to identify the per-patient in-hospital cost of hypoglycemia.

\section{Statistical analyses}

Baseline characteristics were reported using descriptive statistics. To assess LoS, a general linear model was used, adjusting for covariates and testing for potential differences between the groups. Stepwise exclusion of nonsignificant covariates in the statistical model allowed us to gauge their impact on LoS. The statistical model was also used to identify potential risk factors impacting on LoS. Analysis of all-cause mortality was performed via logistic regression, adjusting for the covariates (described previously). A sensitivity analysis comprising a zerotruncated negative binomial and Cox regression was performed to understand the influence of missing data on the choice of model for LoS. A $\gamma$ model with a logarithmic link function was used to estimate the cost associated with LoS. Stepwise backward elimination was used in all regression models. All statistical tests were two-sided; significance was defined as 5\%. Age, gender, and diabetes type were included in all models irrespective of significance. All statistical analyses were carried out using SAS V.9.3 and SAS Enterprise guide V.5.1 software (Cary, USA).

The protocol (13_063R) described above was approved by the Independent Scientific Advisory Committee (ISAC). ISAC is independent of the Medicines and Healthcare Products Regulatory Agency and the CPRD.

\section{RESULTS}

\section{Hypoglycemia as a primary diagnosis (retrospective} cohort study)

A total of 1131 patients, accounting for 1490 hospitalizations, contributed to the retrospective cohort study (297 with type 1 and 834 with type 2 diabetes), all with a primary diagnosis of hypoglycemia. Baseline demographics evaluated at hospitalization $($ mean $\pm \mathrm{SD})$ were age $57.1 \pm 21.5$ years, BMI $(\mathrm{n}=167) \quad 25.2 \pm 5.1 \mathrm{~kg} / \mathrm{m}^{2}$, $\mathrm{HbA}_{1 \mathrm{c}}(\mathrm{n}=215) \quad 8.8 \pm 2.0 \% \quad(72.7 \pm 21.8 \mathrm{mmol} / \mathrm{mol})$, and Charlson comorbidity index score $(n=404) 3.5 \pm 2.1$ for patients with type 1 diabetes, and age $75.7 \pm 12.5$ years, BMI $(\mathrm{n}=482) 28.1 \pm 6.4 \mathrm{~kg} / \mathrm{m}^{2}, \mathrm{HbA}_{1 \mathrm{c}}(\mathrm{n}=683) 8.5 \pm 2.0 \%$ $(69.4 \pm 21.9 \mathrm{mmol} / \mathrm{mol})$, and Charlson comorbidity index score $(\mathrm{n}=1086) 4.0 \pm 2.2$ for patients with type 2 diabetes. Population characteristics at index date are shown in table 1. Eighty-six per cent of patients were admitted to hospital via the accident and emergency department, while $10 \%$ were transferred to hospital via a general practitioner.

The mean LoS for patients with type 1 and 2 diabetes was 5.46 (95\% CI 4.62 to 6.45$)$ and 5.04 (95\% CI 4.46 to 5.71) days, respectively. The estimated mean LoS for patients with hypoglycemia as a primary diagnosis is shown in table 2. There was no significant difference in LoS for patients with type 1 and 2 diabetes. $\mathrm{HbA}_{1 \mathrm{c}}$, Charlson comorbidity index score, insulin regimen, smoking status, and BMI did not influence the LoS. Gender, OAD regimen, and age at first admission to hospital for hypoglycemia had a significant influence on LoS, with older female patients and those treated with sulfonylureas/glinides remaining longer in hospital. Each year increase in age increased LoS by $1.02 \%$ (95\% CI 1.02 to 1.02$), p<0.0001$. Probability values for risk covariates in the final regression model are shown in table 2. We identified the cost for $1454(97.6 \%)$ of the total number of admissions to hospital. The mean total estimated expenditure per hospital admission for hypoglycemia was $£ 1034$ (95\% CI 855 to 1253). There was no difference in cost between patients with type 1 and 2 diabetes. In the regression analysis, age $(p<0.001)$, insulin regimen $(\mathrm{p}=0.009)$, and OAD regimen $(\mathrm{p}=0.04)$ had a significant interaction with treatment cost. The results of the sensitivity analyses were in agreement with those of the regression models.

Table 1 Population characteristics at index date: hypoglycemia as a primary diagnosis

\begin{tabular}{|c|c|c|c|c|}
\hline & \multicolumn{2}{|c|}{$\begin{array}{l}\text { Type } 1 \text { diabetes } \\
(n=404)\end{array}$} & \multicolumn{2}{|c|}{$\begin{array}{l}\text { Type } 2 \text { diabetes } \\
(n=1086)\end{array}$} \\
\hline & $\mathbf{n}$ & $\%$ & $\mathbf{n}$ & $\%$ \\
\hline $\begin{array}{l}\text { Gender } \\
\text { (male/female) }\end{array}$ & $232 / 172$ & $57.4 / 42.6$ & $542 / 544$ & $49.9 / 50.1$ \\
\hline \multicolumn{5}{|l|}{ Smoking status } \\
\hline Never smoked & 75 & 18.6 & 137 & 12.6 \\
\hline Ever smoked & 329 & 81.4 & 949 & 87.4 \\
\hline \multicolumn{5}{|l|}{ Insulin regimen } \\
\hline Basal only & 20 & 5.0 & 196 & 18.0 \\
\hline Bolus only & 19 & 4.7 & 19 & 1.7 \\
\hline Basal-bolus & 152 & 37.6 & 122 & 11.2 \\
\hline Premix & 167 & 41.3 & 561 & 51.7 \\
\hline Other ${ }^{*}$ & 46 & 11.4 & 188 & 17.3 \\
\hline \multicolumn{5}{|l|}{ OAD regimen } \\
\hline Metformin & 11 & 2.7 & 163 & 15.0 \\
\hline $\begin{array}{l}\text { Sulfonylurea } \\
+ \text { glinides }\end{array}$ & 3 & 0.7 & 45 & 4.1 \\
\hline Other OAD & 109 & 27.0 & 294 & 27.1 \\
\hline No OAD & 281 & 69.6 & 584 & 53.8 \\
\hline
\end{tabular}


Table 2 Hypoglycemia as a primary diagnosis. Estimated mean LoS (days).

\begin{tabular}{|c|c|c|c|c|}
\hline & $\begin{array}{l}\text { Mean } \\
\text { LoS } \\
\text { (days) }\end{array}$ & $\begin{array}{l}95 \% \mathrm{Cl} \\
\text { (lower) }\end{array}$ & $\begin{array}{l}95 \% \mathrm{Cl} \\
\text { (upper) }\end{array}$ & p Value* \\
\hline Overall & 5.25 & 4.63 & 5.95 & \\
\hline \multicolumn{5}{|l|}{ Gender } \\
\hline Female & 5.57 & 4.85 & 6.40 & \multirow[t]{2}{*}{0.0482} \\
\hline Male & 4.95 & 4.31 & 5.67 & \\
\hline \multicolumn{5}{|l|}{ Diabetes type } \\
\hline 1 & 5.46 & 4.62 & 6.45 & \multirow[t]{2}{*}{0.3103} \\
\hline 2 & 5.04 & 4.46 & 5.71 & \\
\hline \multicolumn{5}{|l|}{ OAD regimen } \\
\hline Metformin & 4.53 & 3.73 & 5.50 & \multirow[t]{4}{*}{0.0275} \\
\hline $\begin{array}{l}\text { Sulfonylureas/ } \\
\text { glinides }\end{array}$ & 6.07 & 4.34 & 8.49 & \\
\hline Other OAD & 4.86 & 4.24 & 5.57 & \\
\hline No OAD & 5.68 & 5.10 & 6.32 & \\
\hline \multicolumn{5}{|c|}{$\begin{array}{l}\text { *p Values for categorical variables retained in the generalized } \\
\text { linear model analysis of potential risk factors. } p \text { Values indicate } \\
\text { the level of significance for each variable's influence on the } \\
\text { outcome. } \\
\text { LoS, length of stay; OAD, oral antidiabetic drug. }\end{array}$} \\
\hline
\end{tabular}

\section{Inpatient hypoglycemia (matched retrospective cohort study)}

For the second analysis, the matched cohort consisted of 1079 pairs of hospitalized patients (212 and 867 pairs of patients with type 1 and 2 diabetes, respectively). Baseline characteristics $($ mean $\pm \mathrm{SD})$ were age $66.5 \pm 16.1$ years, BMI $(\mathrm{n}=755) \quad 27.9 \pm 6.0 \mathrm{~kg} / \mathrm{m}^{2}, \mathrm{HbA}_{1 \mathrm{c}}(\mathrm{n}=641) \quad 9.6 \pm 2.1 \% \quad(81.4$ $\pm 22.6 \mathrm{mmol} / \mathrm{mol}$ ), and Charlson comorbidity index score $(\mathrm{n}=1079) 4.0 \pm 2.3$ in patients who experienced hypoglycemia during hospitalization, and age $63.1 \pm 16.1$ years, BMI (n=734) $29.3 \pm 6.5 \mathrm{~kg} / \mathrm{m}^{2}, \mathrm{HbA}_{\mathrm{lc}}(\mathrm{n}=626) \quad 9.2 \pm 2.2 \%$ $(77 \pm 24.1 \mathrm{mmol} / \mathrm{mol})$, and Charlson comorbidity index score $(\mathrm{n}=1079) 4.0 \pm 2.5$ for those who did not experience hypoglycemia during hospitalization. Population characteristics at hospitalization for exposed (experienced hypoglycemia during hospital stay) and matched unexposed patients are provided in table 3. Major categories of primary diagnoses are provided in online supplementary table S2.

Exposed patients spent 11.91 (95\% CI 10.96 to 12.94) days in hospital compared with 4.80 (95\% CI 4.41 to 5.23) days for unexposed patients, $\mathrm{p}<0.0001$ (table 4). After multivariate adjustment, age and Charlson comorbidity index score were found to have a significant influence on LoS, $\mathrm{p}<0.05$. Increasing LoS was a function of increasing age in exposed and unexposed patients (figure 1). Exposed patients were more likely to die in hospital compared with unexposed patients (OR 1.439 (95\% CI 1.060 to 1.952$), \mathrm{p}<0.0195)$. Increased age was also associated with a higher risk of all-cause mortality among exposed patients (OR 1.026 (95\% CI 1.015 to $1.038), \mathrm{p}<0.0001)$. Sensitivity analyses confirmed the results of the regression models.

Costs were identified for 1502 (69.6\%) of 2158 admissions. Derived from these, the estimated total average per-patient cost for exposed patients was £2235,

Table 3 Population characteristics at hospitalization: inpatient hypoglycemia

\begin{tabular}{|c|c|c|c|c|}
\hline & \multicolumn{2}{|c|}{$\begin{array}{l}\text { Exposed ( } n=1079 ; \\
\text { with hypoglycemia) }\end{array}$} & \multicolumn{2}{|c|}{$\begin{array}{l}\text { Unexposed ( } \mathrm{n}=1079 ; \\
\text { no hypoglycemia) }\end{array}$} \\
\hline & $\bar{n}$ & $\%$ & $\bar{n}$ & $\%$ \\
\hline \multicolumn{5}{|l|}{ Gender } \\
\hline Male & 567 & 52.5 & 582 & 53.9 \\
\hline Female & 512 & 47.5 & 497 & 46.1 \\
\hline \multicolumn{5}{|l|}{ Diabetes type } \\
\hline 1 & 212 & 19.6 & 212 & 19.6 \\
\hline 2 & 867 & 80.4 & 867 & 80.4 \\
\hline \multicolumn{5}{|l|}{ Smoking status } \\
\hline Never smoked & 115 & 10.7 & 131 & 12.1 \\
\hline Ever smoked & 964 & 89.3 & 948 & 87.9 \\
\hline \multicolumn{5}{|c|}{ Insulin regimen at admission } \\
\hline Basal only & 135 & 12.5 & 124 & 11.5 \\
\hline Bolus only & 16 & 1.5 & 21 & 1.9 \\
\hline Basal-bolus & 114 & 10.6 & 124 & 11.5 \\
\hline Premix & 328 & 30.4 & 280 & 25.9 \\
\hline Other* & 486 & 45.0 & 530 & 49.1 \\
\hline \multicolumn{5}{|c|}{ OAD regimen at admission } \\
\hline Metformin & 122 & 11.3 & 138 & 12.8 \\
\hline Sulfonylurea/glinides & 50 & 4.6 & 71 & 6.6 \\
\hline Other & 63 & 5.8 & 83 & 7.7 \\
\hline No OAD & 844 & 78.2 & 787 & 72.9 \\
\hline
\end{tabular}

*Any insulin regimen that cannot be readily classified as basal only, bolus only, basal-bolus, or premix.

$\mathrm{OAD}$, oral antidiabetic drug. 
Table 4 Inpatient hypoglycemia. Estimated mean LoS (days).

\begin{tabular}{lllll}
\hline & $\begin{array}{l}\text { Mean } \\
\text { LoS } \\
\text { (days) }\end{array}$ & $\begin{array}{l}\text { 95\% } \\
\text { Cl } \\
\text { (lower) }\end{array}$ & $\begin{array}{r}\text { 95\% Cl } \\
\text { (upper) }\end{array}$ & p Value* \\
\hline $\begin{array}{l}\text { Hypoglycemia } \\
\text { Exposed (with }\end{array}$ & 11.91 & 10.96 & 12.94 & $<0.0001$ \\
hypoglycemia) & & & & \\
Unexposed (no & 4.80 & 4.41 & 5.23 & \\
hypoglycemia) & & & & \\
Gender & & & & 0.0796 \\
Female & 7.91 & 7.27 & 8.61 & \\
Male & 7.23 & 6.65 & 7.86 & \\
Diabetes type & & & & 0.4907 \\
1 & 7.75 & 6.85 & 8.76 & \\
2 & 7.38 & 6.94 & 7.84 & \\
CCl score & & & & 0.0033 \\
1 & 6.23 & 5.50 & 7.07 & \\
2 & 8.11 & 6.94 & 9.49 & \\
3 & 7.29 & 6.46 & 8.22 & \\
4 & 8.79 & 7.67 & 10.08 & \\
$5+$ & 7.63 & 6.94 & 8.40 & \\
\hline
\end{tabular}

${ }^{*} p$ Values for categorical variables retained in the generalized linear model analysis of potential risk factors. $p$ Values indicate the level of significance for each variable's influence on the outcome.

$\mathrm{CCl}$, Charlson comorbidity index; LoS, length of stay.

compared with $£ 1591$ for unexposed patients). This difference between groups remained in a multivariate adjusted $\gamma$ model with log link; 38.7\% (95\% CI 29.6 to 48.5), $\mathrm{p}<0.0001$.

\section{DISCUSSION}

To the best of our knowledge, this is the first study to estimate, in a nationally representative population, the resource implications and expenditure associated with outpatient and inpatient hypoglycemia. The results of

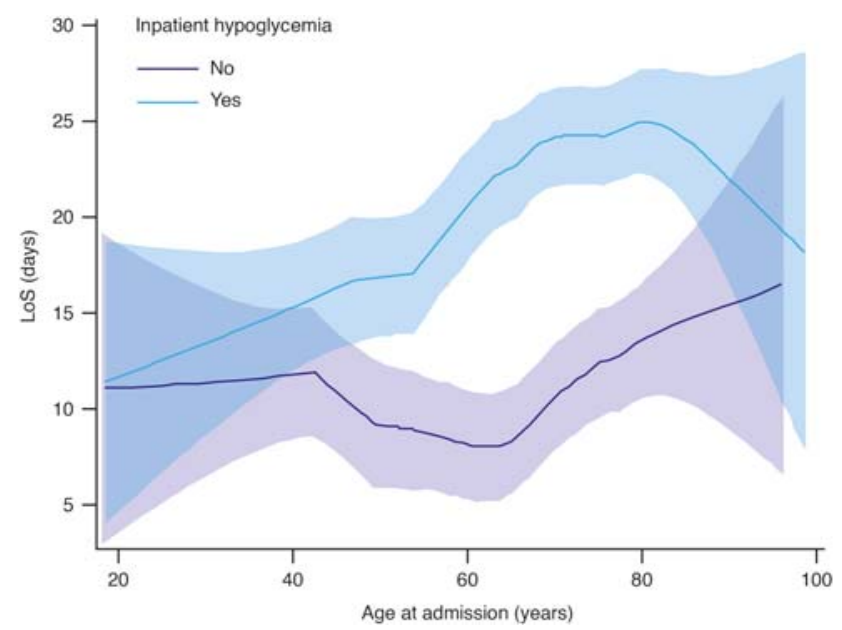

Figure 1 Inpatient hypoglycemia. Log transformation of length of stay (LoS) by age for exposed and unexposed patients. Curves and $95 \% \mathrm{Cl}$ (shaded areas) are fitted by a Loess curve with a smoothing parameter 0.5 . our longitudinal analyses reveal the substantial economic burden of treating patients following a hypoglycemic event, and confirm that experiencing hypoglycemia in hospital increases the mean LoS, risk of all-cause mortality, and associated treatment costs. Despite the best efforts of patients and physicians, not all hypoglycemic events can be avoided; however, we can identify risk factors for patients who are admitted to hospital. In both of our cohorts, age was associated with an increased LoS, and in the matched retrospective study (inpatient hypoglycemia) age was also linked with an increased risk of mortality; therefore, it may be pertinent to develop strategies targeting these patients through dose adjustment. This would be in keeping with the move toward individualized patient care, particularly for patients with type 2 diabetes, advocated by the ADA/ European Association for the Study of Diabetes (EASD) ${ }^{18}$ In addition, targeting the most resource intensive patients could help reduce healthcare expenditure. The cost analyses in our study highlight the economic burden of hypoglycemia on healthcare payers.

The greatest increases in LoS among exposed patients in the matched retrospective cohort study were observed in patients with primary diagnoses of cerebral infarction, heart failure, and acute renal failure. We speculate that the relatively greater severity of such diagnoses, compared with other primary diagnoses, makes these patients particularly vulnerable to the consequences of hypoglycemia on their general health status.

The absence of randomization of patients in exposure groups is an inherent problem in observational studies. Although we cannot be certain that hypoglycemia is the determining factor for LoS or risk of mortality in the inpatient analysis, matching the two patient groups on primary diagnosis and other background variables reduced confounding. In the retrospective cohort study, although all patients had diabetes and were receiving insulin therapy, we cannot exclude the possibility that some hypoglycemic episodes may have been due to other causes, for example, sepsis or alcoholism, rather than medication. Likewise, for the matched retrospective cohort study, we do not have data on insulin or OAD prescriptions during admission, which limits our ability to confirm that the inpatient hypoglycemic episodes were related to medication. Confounding in the matched retrospective cohort study may have occurred due to patients not being matched on the Charlson comorbidity index score. Another limitation of this study is that there are no blood glucose measurements available to confirm hypoglycemia. Despite this, we believe that the classifications of events are likely to be accurate, as the recordings were made by experienced healthcare professionals. Miscoding of hypoglycemia events is possible, and it may not have been the reason for hospitalization in all patients. In these cases, it is conceivable that the patient would have been admitted anyway for an underlying symptom. However, since hypoglycemic events were recorded by healthcare professionals, this 
was unlikely to be a major source of confounding. In the inpatient study, the time of the hypoglycemic episode during the hospital stay was not recorded. This prevents us from estimating the proportion of increased LoS that can be attributed to hypoglycemic events and any resulting economic consequences. As with other register-based studies, we cannot be sure that patients had a continuous use of insulin after the second prescription. ${ }^{19}$ This study did not record patients' history of outpatient hypoglycemia, and thus it is possible that there is an overlap between our study populations. Our analysis does not permit causality to be ascribed, but it does enable derivation of an associated cost between hypoglycemia and hospitalization. Costs were derived from a subset of patients for both the retrospective and matched retrospective cohorts; however, since the subsets were large and $\operatorname{LoS}$ is the main driver of costs, these estimates should be representative of the full data sets.

Although this study uses data from England, we anticipate that similar results would be obtained in other countries. A major strength of our analysis is that UK databases, such as the CPRD, provide long-term coverage of patients' healthcare records.

In summary, hospitalization of people with diabetes following a hypoglycemic episode leads to significant expenditure and use of healthcare resources. Furthermore, inpatients who experience hypoglycemia are likely to have a greatly increased LoS and are at a higher risk of dying in hospital. Reducing the incidence of hypoglycemia in the community and in hospital is medically and economically important, with the potential to generate significant cost savings for healthcare providers at a national level.

Acknowledgements The authors acknowledge medical writing assistance from Paul Tisdale, PhD, and editorial support from Gabrielle Parker, BSc, of Watermeadow Medical, UK (sponsored by Novo Nordisk). The authors would like to thank Søren Rud Kristensen, research fellow, Manchester Centre of Health Economics Institute of Population Health, University of Manchester, for assisting in transforming, grouping, and interpreting HRG cost data. The data from the inpatient hypoglycemia analysis have previously been published as an abstract and oral presentation at ADA 2014.

Contributors PM and ME contributed to the conception and design of the article, analysis plan, and data interpretation. JJ contributed to the statistical analysis, data interpretation, and writing of the article. BLT and MW contributed to the study design, data analysis, and data interpretation.

Competing interests PM has received research funding from Novo Nordisk, Sanofi, BMS, AZ, Lilly, GSK, and Takeda. BLT and MW are employees and shareholders of Novo Nordisk A/S. JJ is a consultant for, and shareholder of, Novo Nordisk A/S. ME has received honoraria as an advisory panel member and speaker from Novo Nordisk, Sanofi Aventis, Novartis, and MSD.

Provenance and peer review Not commissioned; externally peer reviewed. Data sharing statement No additional data are available.

Open Access This is an Open Access article distributed in accordance with the Creative Commons Attribution Non Commercial (CC BY-NC 4.0) license, which permits others to distribute, remix, adapt, build upon this work noncommercially, and license their derivative works on different terms, provided the original work is properly cited and the use is non-commercial. See: http:// creativecommons.org/licenses/by-nc/4.0/

\section{REFERENCES}

1. Fidler C, Elmelund CT, Gillard S. Hypoglycemia: an overview of fear of hypoglycemia, quality-of-life, and impact on costs. J Med Econ 2011;14:646-55.

2. Budnitz DS, Lovegrove MC, Shehab N, et al. Emergency hospitalizations for adverse drug events in older Americans. N Engl $J$ Med 2011:365:2002-12.

3. Leese GP, Wang J, Broomhall J, et al. Frequency of severe hypoglycemia requiring emergency treatment in type 1 and type 2 diabetes: a population-based study of health service resource use. Diabetes Care 2003;26:1176-80.

4. Tan HK, Flanagan D. The impact of hypoglycaemia on patients admitted to hospital with medical emergencies. Diabet Med 2013;30:574-80.

5. Turchin A, Matheny ME, Shubina M, et al. Hypoglycemia and clinical outcomes in patients with diabetes hospitalized in the general ward. Diabetes Care 2009;32:1153-7.

6. Garg R, Hurwitz S, Turchin A, et al. Hypoglycemia, with or without insulin therapy, is associated with increased mortality among hospitalized patients. Diabetes Care 2013;36:1107-10.

7. Hsu PF, Sung SH, Cheng HM, et al. Association of clinical symptomatic hypoglycemia with cardiovascular events and total mortality in type 2 diabetes: a nationwide population-based study. Diabetes Care 2013;36:894-900.

8. Johnston SS, Conner C, Aagren M, et al. Evidence linking hypoglycemic events to an increased risk of acute cardiovascular events in patients with type 2 diabetes. Diabetes Care 2011;34:1164-70.

9. Johnston SS, Conner C, Aagren M, et al. Association between hypoglycaemic events and fall-related fractures in Medicare-covered patients with type 2 diabetes. Diabetes Obes Metab 2012;14:634-43.

10. Zoungas S, Patel A, Chalmers J, et al. Severe hypoglycemia and risks of vascular events and death. N Engl J Med 2010;363:1410-18.

11. Farmer AJ, Brockbank KJ, Keech ML, et al. Incidence and costs of severe hypoglycaemia requiring attendance by the emergency medical services in South Central England. Diabet Med 2012;29:1447-50.

12. Hammer M, Lammert M, Mejias SM, et al. Costs of managing severe hypoglycaemia in three European countries. J Med Econ 2009;12:281-90.

13. International Diabetes Federation. IDF Diabetes Atlas. 6th edn. IDF Diabetes Atlas, 2013. http://www.idf.org/diabetesatlas

14. Chen YC, Wu JC, Haschler I, et al. Academic impact of a public electronic health database: bibliometric analysis of studies using the general practice research database. PLOS ONE 2011;6:e21404.

15. Seaquist ER, Anderson J, Childs B, et al. Hypoglycemia and diabetes: a report of a workgroup of the American diabetes association and the endocrine society. Diabetes Care 2013;36:1384-95.

16. Khan NF, Perera R, Harper S, et al. Adaptation and validation of the Charlson Index for Read/OXMIS coded databases. BMC Fam Pract 2010;11:1.

17. Health and Social Care Information Centre. Health and Social Care Information Centre. 2014. http://www.hscic.gov.uk/article/2021/ Website-Search?q=hrg+grouper+software\&go=Go\&area=website

18. Inzucchi SE, Bergenstal RM, Buse JB, et al. Management of hyperglycemia in type 2 diabetes: a patient-centered approach: position statement of the American Diabetes Association (ADA) and the European Association for the Study of Diabetes (EASD). Diabetes Care 2012;35:1364-79. Erratum in Diabetes Care 2013;36:490.

19. Carstensen B, Witte DR, Friis S. Cancer occurrence in Danish diabetic patients: duration and insulin effects. Diabetologia 2012;55:948-58. 